Journal of Computer Science 7 (2): 250-254, 2011

ISSN 1549-3636

(C) 2011 Science Publications

\title{
Computer Aided Diagnosis System for Stone Detection and Early Detection of Kidney Stones
}

\author{
${ }^{1}$ Ms.P.R. Tamilselvi and ${ }^{2}$ Dr.P. Thangaraj \\ ${ }^{1}$ Department of Computer Technology, Kongu Engineering College, \\ Perundurai, 638 052, Tamilnadu, India \\ ${ }^{2}$ Department of Computer Science and Engineering, \\ Bannari Amman Institute of Technology Sathyamangalam, Tamilnadu, India
}

\begin{abstract}
Problem statement: Most of the previous study in diagnosis of kidney stone identifies a mere presence or absence of the stones in the kidney. However proposal in our study even present an early detection of kidney stones which helps to change the diet conditions and prevent the formation of stones. Approach: The study presented a scheme for ultrasound kidney image diagnosis for stone and its early detection based on improved seeded region growing based segmentation and classification of kidney images with stone sizes. With segmented portions of the images the intensity threshold variation helps in identifying multiple classes to classify the images as normal, stone and early stone stages. The improved semiautomatic Seeded Region Growing (SRG) based image segmentation process homogeneous region depends on the image granularity features, where the interested structures with dimensions comparable to the speckle size are extracted. The shape and size of the growing regions depend on this look up table entries. The region merging after the region growing also suppresses the high frequency artifacts. The diagnosis process is done based on the intensity threshold variation obtained from the segmented portions of the image and size of the portions compared to that of the standard stone sizes (less than $2 \mathrm{~mm}$ absence of stone, 2-4 mm early stages and $5 \mathrm{~mm}$ and above presence of kidney stones). Results: The parameters of texture values, intensity threshold variation and stones sizes are evaluated with experimentation of various Ultrasound kidney image samples taken from the clinical laboratory. The texture extracted from the segmented portion of the kidney images presented in our study precisely estimate the size of the stones and the position of the stones in the kidney which was not done in the earlier studies. Conclusion: The integrated improved SRG and classification mechanisms presented in this study diagnosis the kidney stones presence and absence along with the early stages of stone formation.
\end{abstract}

Key words: Ultrasound images, kidney stones, early detection, image segmentation, Seeded Region Growing (SRG), stone detection, Intensity threshold

\section{INTRODUCTION}

The study has focused on the kidney image segmentation and diagnosis for stone detection and absence in the ultrasound images. First, the kidney moves within the patient owing to breathing, at several centimeters of amplitude (Van Sornsen de Koste et al., 2006) and irrigation liquids are used during the operation. These two constraints have a direct effect on image quality. Moreover, it is not uncommon to break some optical fibers, resulting in black dots on the image. Second, kidney stones have demonstrated different chemical compositions, resulting in different shapes, colors and textures (Leusmann, 1991). Finally, the system must be fast enough to work over the laser shooting rate. Though medical image segmentation is a very active domain (Duncan and Ayache, 2000; Rao, 2004), few studies have examined this particular subject. Most of them have focused on MRI (Vivier et al., 2008; Makni et al., 2009), CT scan or ultrasound images (Sridhar et al., 2002) San Jose Estepar et al., 2009). Except for laparoscopic images (Voros et al., 2006), only a few researches have directly examined video images. In this study, integrated image processing (with improved image segmentation and diagnosis) scheme performs the function semi automatically, without the need for an interaction from the user to diagnosis kidney stones presence, absence and early detection

Corresponding Author: Ms.P.R. Tamilselvi, Department of Computer Technology, Kongu Engineering College, Perundurai, 638 052, Tamilnadu, India 
from Ultrasound images. The initial stage has been presented and its precision, robustness and speed have been examined.

Integrated image segmentation and diagnosis of ultrasound kidney images: The ultrasound image is non invasive among various imaging modalities with features like low cost imaging, minimal scan time, flexible operation and reduced exposure to harmful radiation. The segmentation and classification technique evaluate the features of the image acquired and display result for kidney stones diagnosis.

Ultrasound kidney image segmentation: Semi automated SRG algorithm for kidney image segmentation are comprises of three steps i.e., seed point selection, seed region growing and optimal threshold value. A seed is a perquisite and need to automatically select a seed point replacing the selection through user interaction. With the given seed SRG can start to grow, but a threshold value has to be determined to only cover the reasonable pixels.

The local variance and mean ratio of the granularity in the fully developed ultrasound speckle kidney image is used as the measured parameter for seed point selection. According to this parameter, it is possible to decide whether the processed pixel is within homogeneous region or not. In general, if the local variance to mean ratio is larger than that of speckle, then the corresponding pixel can be considered as a resolvable object. Otherwise, it belongs to a homogeneous region. The shape of speckle pattern and average speckle size varies at different locations of stored images. Therefore, it is highly desirable to arbitrarily defined shape and size of the homogeneous regions for smoothing. This is achieved through the region growing procedure, which effectively fits the grown region to the homogeneous area without imposing any shape constraint. The region growing procedure employs a look-up table consisting of statistical bounds for different values of local statistics.

The aim of region-based segmentation techniques is to extract the homogeneous seed points from the ultrasound filtered image. Region growing technique is better in noisy images, where borders are extremely difficult to detect such as ultrasound medical images. For region growing method homogeneity is an important property, which can be based on gray-level, shape, model. For region- based segmentation, the basic requirement is to satisfy the region similarity in the kidney image.
The Proposed semi-automatic seeded region growing algorithm for ultrasonic kidney images:

- Choose a window sized $(2 \mathrm{k}+1) \times(2 \mathrm{k}+1)$ being centered at (i, j) for seed point

- Generate the look up table for local statistics for each pixel:

- Calculate the homogeneity

- Calculate the statistical similarity bound

- Implement region growing for every pixel:

- Each image pixel is taken as a seed pixel

- Store the neighboring pixel information for every seed point

- Grow region from the seed point according to the statistical similarity criterion

- Implement region merging:

- Labeling the each region with a unique number

- Store the neighboring region information for every seed region

- Merge the neighboring region according to the merging criteria with the seed region

- Update the segmented image output

Image diagnosis: Image diagnosis is based on the texture of the segmented portion of the images compared to that of standard benchmarked kidney image texture values. Texture extraction is the process of quantifying the texture patterns within a specified neighborhood of size $\mathrm{M}$ by $\mathrm{N}$ pixels around a pixel of interest. There mainly exist four categories of texture analysis, namely, structural, statistical, model-based and transform-based approaches. In our study, two sets of texture features, namely statistical i.e., first and second order coefficients and structural i.e., Haralick texture descriptors, are used separately to compare their performances in characterizing type of renal calculi.

The Ultrasound kidney image is $x(i, j)$ ' $T_{R}$ ' is the total number of pixels in kidney region " $\mathrm{R}$ ". The first order gray level statistical features mean Pixel Rate (Mn), Dispersion Pixel Rate (Dn), Variance Pixel Rate (Vn), Energy Joules (En) and Entropy Rate (Ep) are estimated as:

$$
\begin{aligned}
& \mathrm{Mn}=\frac{1}{\mathrm{~T}_{\mathrm{R}}} \sum \sum_{\mathrm{Rij} \in \mathrm{R}}[\mathrm{x}(\mathrm{i}-\mathrm{k}, \mathrm{j}-\mathrm{l})] \\
& \mathrm{Dn}=\sum \sum_{\mathrm{Rij} \in \mathrm{R}}[\mathrm{x}(\mathrm{i}-\mathrm{k}, \mathrm{j}-\mathrm{l})-\operatorname{Mean}(\mathrm{k}, \mathrm{l})]
\end{aligned}
$$




$$
\begin{aligned}
& \text { Vn }=\frac{1}{T_{R}} \sum \sum_{\text {RijeR }}[x(i-k, j-l)-\text { Mean }(k, l)]^{2} \\
& \text { En }=\frac{1}{T_{R}} \sum \sum_{R i j \in R}[x(i-k, j-l)]^{2} \\
& E p=\sum_{x y} x(i, j) \log I(k, l)
\end{aligned}
$$

If the intensity values $I(k, l)$ of $x(i, j)$ are arranged in ascending order, then:

Median $=\mathrm{I}(\mathrm{k}, \mathrm{l})$ of $\frac{\mathrm{N}+1}{2}$ pixel

In case of even $\mathrm{N}$, the median value is estimated by finding the average of two middle values. The integration of the image segmentation and classification is done with intensity threshold variation associated to the texture features for the characterization of region from images. The two methods used in this study for texture description are statistical and structural. The Spatial Gray Level Dependence Method (SGLDM) is adopted for statistical texture description. All known visually distinct texture pairs can be discriminated using above method. These statistical features of second order are computed in a two step process. The first step delivers the co-occurrence matrices containing the element $P_{k}(i, j)$. Each (i, j)th entry of the matrices represents the probability of going from pixel with gray level (i) to another with a gray level (j) under a predefined newly suggested angles, $0,30,60,90,120$ and 150 degrees. Based on the co-occurrence matrices texture features are estimated to diagnosis the stones presence, absence and early stages in the kidney images.

\section{MATERIALS AND METHODS}

Experimentation of kidney image segmentation and classification: Ultrasound kidney images with normal and stones of various sized is obtained from the medical laboratory. The images acquired by ultrasound scanner were processed using discriminative features. The edges of the stones were clearly observed on the segmented portion of the images with classification indicate the size of the stone presented. The images with segmented classification find the average, mean and median values of intensities of the pixels in the image.

The seed is the starting point of the region growth. The position of the seed must be inside the calculus in the kidney image. The segmented image is then a binary image representing the inside and the outside of the calculus on the kidney image. The similarity criteria of the region growing algorithm allows to differentiating the calculus from the rest of the image. Hence, it is possible to study with calculi of different colors and textures to ensure a proper functioning in most of the clinical scenario. The similarity criterion gives a score representing the similarity between the region already found and the pixel or group of pixels examined. The stopping criterion is defined as a threshold on the value of the similarity criterion. Its value must be optimized to give the best segmentation possible.

The experimentation conducted for these parameters were set after a study made on a dataset composed of more than 150 images. The solution for accurate results is to preset the algorithm with the found parameters and to allow the medicos to adjust the parameters around this preset at the beginning of the diagnosis. The standard results obtained from the manual detection of kidney stones from the ultrasound images have been traced by expert and urologist who is even prone to variability. The proposed automated study of integrated segmentation and diagnosis provides precise stone detection in the kidney for any number of ultrasound kidney images. The detection rate for the red spot is nearly $95 \%$, the impact of specular reflections on the surface of the kidney images and eventual blood drops flowing through the kidney was examined with noise preprocessing stages (Rizon et al., 2005).

\section{RESULTS}

Performance evaluation of kidney stone diagnosis and early detection: The improved semiautomatic region growing algorithm was developed and set up for the most frequent usable clinical scenario. Manual segmentations were made by the medical experts of the clinical laboratory on real ultrasound images to establish the actual position and a quantitative error measurement. The adaptation of Local Homogeneity Criterion was used in gray ultrasound kidney images. The stopping criterion was a intensity threshold variation of 15, shown in Fig. 1 (stone detected images) and the window size was set to $6 \times 6$ pixels.

The manual initialization obtain good results on the validation ultrasound kidney image classification and diagnosis of the stone presence and early stages composed of images coming from different clinical situations. The stone size formed in normal patient is less than $2 \mathrm{~mm}$ however with size of $5 \mathrm{~mm}$ and above 


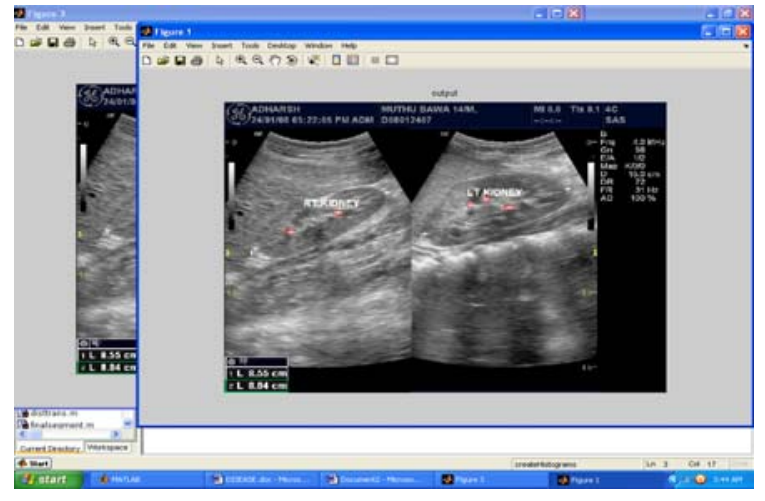

Fig. 1: Detection of kidney stone formation in US kidney images

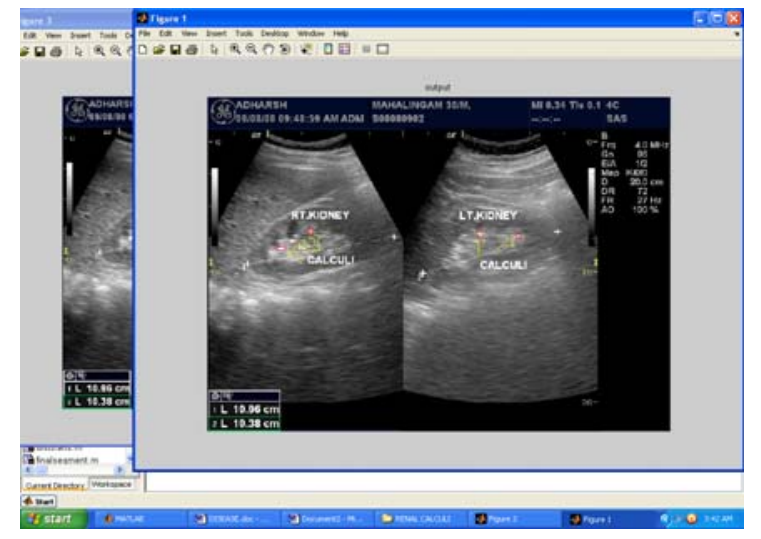

Fig. 2: Early stages of stone formation in US kidney images

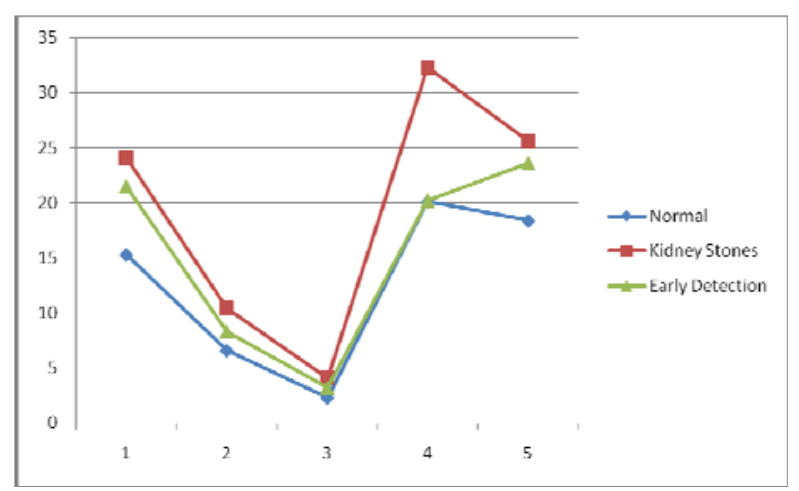

Fig. 3: Comparison of extracted features

cause serious issue to the kidney. The size of the stones in the kidney between 3-4 mm is classified as early stages of stone formation in the kidney (Fig. 2).

The tabulation show in table1 (Fig. 3) depicts the effect of proposed method in diagnosing the US kidney
Table .1: Extracted features from US kidney images

\begin{tabular}{llllll}
\hline Kidney images & $\mathrm{Mn}$ & $\mathrm{Dn}$ & $\mathrm{Vn}$ & $\mathrm{En}$ & $\mathrm{Ep}$ \\
\hline Normal & 15.3 & 6.6 & 2.3 & 20.1 & 18.4 \\
Kidney stones & 24.1 & 10.4 & 4.1 & 32.3 & 25.6 \\
Early detection & 21.5 & 8.3 & 3.2 & 20.2 & 23.6 \\
\hline
\end{tabular}

images for its stone detection and early stages of stone formation using various texture features. The study presented in this study utilized improved SRG for segmentation identifies the various intensity threshold segments of the ultrasound kidney images.

\section{DISCUSSION}

With variant of threshold greater than 15 , texture features and size of the stones and segmented portions define the images to diagnosis the presence, absence and early stages of the stone in the kidney. The proposed integration improved SRG and classification is fast enough to run at a frame rate of $20 \mathrm{~Hz}$ and integrated in an automated system to sweep the surface of kidney stones and remove them by remedy measures such as sound shocks to fragment the stones to negligible pieces.

\section{CONCLUSION}

The study presented in the study diagnosis the kidney stone detection and early stages of formation with integrated image segmentation and classification. The segmentation process is carried out with improved SRG for identifying the intensity threshold variation. The texture extracted from the segmented portions is mapped to the size of the pixel squares with its size magnitude. The size and texture property of the segmented image evaluate the absence and presence of kidney stones. The experimentation is conducted to easy and effective diagnosis of stones and its early stages from ultrasound image samples taken from various patients. The parameters for diagnosing and classifying the US kidney stone images are identified and analyzed. The quality features describe the kidney stone size and its position is made viable. There has been significant difference observed in many parameters of each type of renal calculi. It is required to analyze different sizes of the kidney stone and based on these numerical values of features it is highly feasible to develop a universal reference for kidney stone categories.

\section{ACKNOWLEDGMENT}

The Researchers are thankful to Adharsh scan centre, Erode, Tamilnadu, India for providing kidney ultrasound images for the research study. 


\section{REFERENCES}

De Koste, V.S.J.R., S. Senan, C.E. Kleynen, B.J. Slotman and F.J. Lagerwaard, 2006. Renal mobility during uncoached quiet respiration: An analysis of 4DCT scans. Int. J. Radiat. Oncol. Biol. Phys., 64: 799-803. PMID: 16298498

Duncan, J.S. and N. Ayache, 2000. Medical image analysis: Progress over two decades and the challenges ahead. IEEE Trans. Patt. Anal. Machine Intell., 22: 85-106. DOI: 10.1109/34.824822

Estepar, R.S.J., C.F. Westin and K.G. Vosburgh, 2009. Towards real time 2D to 3D registration for ultrasound-guided endoscopic and laparoscopic procedures. Int. J. Comput. Assist Radiol. Surg., 4: 549-560. PMID: 20033331

Leusmann, D.B., 1991. A classification of urinary calculi with respect to their composition and micromorphology. Scand J. Urol. Nephrol., 25: 141-150. PMID: 1871560

Makni, N., P. Puech, R. Lopes, A.S. Dewalle and O. Colot et al., 2009. Combining a deformable model and a probabilistic framework for an automatic 3D segmentation of prostate on MRI. Int. J. Comput. Assist. Radiol. Surg., 4: 181-188. DOI: 10.1007/s11548-008-0281-y

Rao, P.N., 2004. Imaging for kidney stones. World J. Urol., 22: 323-327. DOI: 10.1007/s00345-0040413-0

Rizon, M., H. Yazid, P. Saad, A.Y.M. Shakaff and A.R.S.M. Sugisaka et al., 2005. Object detection using circular hough transform. Am. J. Applied Sci., $\quad 2$ : $1606-1609 . \quad$ DOI: 10.3844/ajassp.2005.1606.1609
Singh, S. and V.R. Singh, 1998. Ultrasonic parameters of renal calculi. Proceedings of 20th Annual International Conference of the IEEE Engineering in Medicine and Biology Society, Oct. 29-1 Nov., Hong Kong , China, pp: 862-865. DOI: 10.1109/IEMBS.1998.745574

Sridhar, S., N. Kumaravel and K.S. Easwarakumar, 2002. Segmentation of renal calculi in ultrasound images. Med. Inform. Internet. Med., 27: 229-236. PMID: 12745904

Staib, L.H. and J.S. Duncant, 2000. Parametrically deformable contour models. Proceedings of the IEEE Computer Society Conference on Lawrence Computer Vision and Pattern Recognition, June 48, San Diego, CA , USA., pp: 98-103. DOI: 10.1109/CVPR.1989.37834

Vivier, P.H., M. Dolores, I. Gardin, P. Zhang and C. Petitjean et al., 2008. In vitro assessment of a 3D segmentation algorithm based on the belief functions theory in calculating renal volumes by MRI. Am. J. Roentgenol, 191: 127-134. DOI: 10.2214/AJR.07.3063

Voros, S., E. Orvain, P. Cinquin and J.A. Long, 2006. Automatic detection of instruments in laparoscopic images: a first step towards high level command of robotized endoscopic holders. Proceeding of the 1st IEEE/RAS-EMBS International Conference on Biomedical Robotics and Biomechatronics, Feb. 20-22, La Tronche, Pisa, pp: 1107-1112. DOI: 10.1109/BIOROB.2006.1639240 